Wright State University

CORE Scholar

$12-1970$

\title{
Nuclear Magnetic Resonance Investigation of the Metal-to- Semiconductor Transition in Crystalline CdO
}

\author{
R. P. Benedict \\ David C. Look \\ Wright State University - Main Campus, david.look@wright.edu
}

Follow this and additional works at: https://corescholar.libraries.wright.edu/physics

Part of the Physics Commons

\section{Repository Citation}

Benedict, R. P., \& Look, D. C. (1970). Nuclear Magnetic Resonance Investigation of the Metal-toSemiconductor Transition in Crystalline CdO. Physical Review B, 2 (12), 4949-4959.

https://corescholar.libraries.wright.edu/physics/609

This Article is brought to you for free and open access by the Physics at CORE Scholar. It has been accepted for inclusion in Physics Faculty Publications by an authorized administrator of CORE Scholar. For more information, please contact library-corescholar@wright.edu. 


\title{
Nuclear Magnetic Resonance Investigation of the Metal-to- Semiconductor Transition in Crystalline $\mathrm{CdO}^{*} *$
}

\author{
R. P. BENEDICT \\ Air Force Institute of Technology, Wright-Patterson Air Force Base, Dayton, Ohio 45433 \\ AND \\ D. C. LooK \\ Department of Physics, University of Dayton, Dayton, Ohio 45433
}

(Received 25 May 1970)

\begin{abstract}
Measurements of the $\mathrm{Cd}^{113}$ nuclear-spin-lattice relaxation time $\left(T_{1}\right)$ and resonance frequency shift $(K)$ were carried out in several samples of polycrystalline $n$-type CdO over the temperature $(T)$ range $1.4-300^{\circ} \mathrm{K}$, frequency $(\nu)$ range $2-10 \mathrm{MHz}$, and carrier concentration $\left(N_{e}\right)$ range $3.3-44 \times 10^{18}$ $\mathrm{cm}^{-3}$. For $N_{e}>10^{19} \mathrm{~cm}^{-3}$, we find that $T_{1} \propto N_{e}^{-2 / 3} T^{-1}$ and $K \propto N_{e}^{1 / 3}$, showing that the nuclei are interacting with degenerate conduction electrons. Furthermore, the Korringa relationship holds and the calculated electron density at a nucleus is almost the same as that in a free atom, indicating that the electrons are in the host-lattice conduction band. The Hall constant is independent of temperature, also suggesting that there is no separate impurity band. Conversely, for $N_{e}<10^{19} \mathrm{~cm}^{-3}$ none of the above-mentioned relationships holds and we must invoke a model in which the electrons are concentrated near impurity centers, forming an impurity band, with nuclei near the centers experiencing strong contact interactions while nuclei far from the centers experience only indirect interactions through nuclear-spin diffusion. These two groups of nuclei do not maintain a common spin temperature and thus the recovery of the magnetization is nonexponential. Finally, the concentration $(N)$ at which the impurity wave functions no longer form a band is estimated from the Mott criterion to be about $2 \times 10^{18} \mathrm{~cm}^{-3}$, slightly below our lowest-concentration sample. Thus, assuming that $N_{e} \simeq N$, we find that for $N<2 \times 10^{18} \mathrm{~cm}^{-3}$ the impurity wave functions are localized, for $2 \times 10^{18}<N<10^{19} \mathrm{~cm}^{-3}$ the electrons are free to move in an impurity band, and for $N>10^{19} \mathrm{~cm}^{-3}$ the Fermi level crosses into the host-lattice conduction band.
\end{abstract}

\section{INTRODUCTION}

Cadmium oxide is a heavily doped semiconductor that has high conductivity due to a large number of donor impurities which are thought to be either $\mathrm{Cd}$ interstitials or oxygen vacancies. ${ }^{1-4}$ The conductionelectron system generally has been found to be degenerate at low temperatures ${ }^{1,3}$ and has been either degenerate $^{1,3}$ or nondegenerate ${ }^{5}$ at high temperatures, depending on the history of the sample, i.e., sintering time and temperature, carrier concentration, and oxygen pressure. The donor impurities, which contribute the conduction electrons, give rise to an impurity level a few tenths of an $\mathrm{eV}$ below the conduction band. ${ }^{6} \mathrm{An}$ impurity band will form when the concentration $(N)$ is high enough that the electrons are able to move freely (without activation) between donors. This is defined by Mott ${ }^{7}$ as the critical concentration $N_{c} \simeq\left(0.25 / a_{H}\right)^{3}$, where $a_{H}$ is the impurity Bohr radius. At a higher impurity concentration, $N_{c b} \simeq(1 / 4 \pi) a_{H}{ }^{-3}$, the impurity band will overlap the conduction band and the material will exhibit metallic properties. ${ }^{8}$ We will refer to the region $N<N_{c}$ as semiconducting; $N_{c}<N<N_{c b}$, quasimetallic; and $N<N_{c b}$, metallic. Most of the CdO reported in the literature appears to have metallic properties. The purpose of this investigation was to lower the electron concentration in $\mathrm{CdO}$ far enough to determine both $N_{c b}$ and $N_{c}$, an expansion of earlier work on a CdO crystal of concentration greater than $N_{c b} .{ }^{9}$

The types of measurements that were used to study the behavior of $\mathrm{CdO}$ as a function of carrier concentra- tion included nuclear-magnetic-resonance (NMR) experiments and Hall-effect measurements. The two NMR properties, nuclear-spin-lattice relaxation and Knight shift, that were used in this investigation have been used previously to study the semiconductor-to-metal transition in Si and Ge. ${ }^{10,11}$ In these studies, the Knight shift was found to decrease very rapidly when $N$ became less than $N_{c b}$ because the electronic wave functions were no longer uniformly spread throughout the crystal. ${ }^{11}$ This behavior of the Knight shift and the failure of the Korringa product to hold are two of the most sensitive tests of the point at which $N=N_{c b}$.

The impurity concentration of $\mathrm{CdO}$ is very hard to control. The method used to vary the concentration was to sinter various types of $\mathrm{CdO}$ powders in air and oxygen and this resulted in a variation of $N_{e}$ from 3.3 to $44.0 \times 10^{18} \mathrm{~cm}^{-3}$. Nuclear-spin-lattice relaxation time measurements, Knight-shift measurements, and Halleffect measurements, made as a function of magnetic field and temperature, are used to determine the value of $N_{c b}$ and to estimate $N_{c}$ in CdO. Some unusual spindynamic properties, such as nonexponential relaxation, occurred in the region $N<N_{c b}$ and are explained in terms of two groups of nuclei, one of the groups consisting of nuclei close enough to the impurity centers to experience strong contact interactions, and the other group consisting of nuclei far from the centers, experiencing only indirect interactions.

In this paper, to relate the Hall measurements to donor impurity concentrations, we will assume that $N \simeq N_{e}$. Such a situation could arise from the donors 4949 
being singly ionized and the compensation very small, or the donors doubly ionized ${ }^{4}$ and the compensation about $50 \%$. The latter situation is more probable, but since we do not know the compensation ratio, it is difficult to make a clearly better approximation than $N \simeq N_{e}$. Indeed, our conclusions must contain this uncertainty.

\section{THEORY}

\section{Nuclear-Spin-Lattice Relaxation}

The Hamiltonian for the magnetic interaction of an electron with the nucleus may be given as ${ }^{12,13}$

$H=\left(\boldsymbol{\mu}_{1} \cdot \boldsymbol{\mu}_{2} / r^{3}\right)-\left[3\left(\boldsymbol{\mu}_{1} \cdot \mathbf{r}\right)\left(\boldsymbol{\mu}_{2} \cdot \mathbf{r}\right) / r^{5}\right]+\frac{8}{3} \pi \mu_{1} \cdot \mu_{2} \delta(\mathbf{r})$,

where $\boldsymbol{\mu}_{1}$ and $\boldsymbol{\mu}_{2}$ are the two interacting magnetic moments and $r$ is the distance between them. We will consider this Hamiltonian for two different relaxation mechanisms, conduction-electron relaxation and fixed paramagnetic impurity relaxation. In the two cases $\mu_{1}$ is a conduction electron or a paramagnetic ion, respectively, and $\mu_{2}$ is a $\mathrm{Cd}^{113}$ nucleus.

The first relaxation mechanism to be considered is relaxation by degenerate conduction electrons. Since the conduction electrons in $\mathrm{CdO}$ are mainly $s$ state around a $\mathrm{Cd}$ site, ${ }^{9}$ and moreover since $\mathrm{CdO}$ is cubic, the dipole-dipole interaction terms (first two terms) can be neglected..$^{13}$ Using the nuclear Zeeman energy as a zeroth-order Hamiltonian and treating the third term of Eq. (1) with time-dependent perturbation theory, one may arrive at an expression ${ }^{13}$ for the nuclear-spin-lattice relaxation time $T_{1}$. For degenerate electrons (which require the use of Fermi statistics) and $k T \ll E_{F}, T_{1}$ is given by

$$
1 / T_{1}=(64 / 9) \pi^{3} \gamma_{e}^{2} \gamma_{n}^{2} \hbar^{3}\left\langle\left|\phi_{F}(0)\right|^{2}\right\rangle^{2} \rho^{2}\left(E_{F}\right) k T,
$$

where $\gamma_{e}$ and $\gamma_{n}$ are the electronic and nuclear gyromagnetic ratios, respectively, $\hbar$ is Planck's constant divided by $2 \pi,\left\langle\left|\phi_{F}(0)\right|^{2}\right\rangle$ is the electronic probability density at the $\mathrm{Cd}^{113}$ nucleus averaged over the states on the Fermi surface, $\rho\left(E_{F}\right)$ is the electronic density of states at the Fermi level, $k$ is Boltzmann's constant, and $T$ is the absolute temperature. From free-electron theory

$$
\rho\left(E_{F}\right)=\left(3 / 8 \pi^{4}\right)^{1 / 3}\left(m_{e}^{*} / \hbar^{2}\right) N_{e}^{1 / 3} V,
$$

where $N_{e}$ is the carrier concentration and $V$ is the sample volume. Equation (3) is, of course, an approximation since the band is believed to be not exactly parabolic. ${ }^{4}$ Upon combining Eqs. (2) and (3), and using ${ }^{14} \gamma_{e}=$ $1.57 \times 10^{7}$ and $\gamma_{n}=5.93 \times 10^{3}$ (for $\mathrm{Cd}^{113}$ ), we get

$$
1 / T_{1}=6.1 \times 10^{33}\left(m_{e}{ }^{*}\right)^{2}\left[\left\langle\left|\phi_{F}(0)\right|^{2}\right\rangle\right]^{2} V^{2} N_{e}{ }^{2 / 3} T .
$$

Thus, for the case of degenerate conduction electrons, $T_{1} \propto N_{e}^{-2 / 3} T^{-1}$. If nondegenerate electrons are con- sidered $^{13}$ (using Maxwell-Boltzmann statistics), $T_{1} \propto$ $N^{-1} T^{-1 / 2}$. For both cases, $T_{1}$ is independent of frequency.

The second applicable relaxation mechanism is the coupling between a paramagnetic impurity and the nucleus of interest. Here the scalar contact term is not effective, since $r \neq 0$, and therefore the dipolar coupling terms are the most important. There are two processes involved in paramagnetic impurity relaxation, a strong direct coupling to nuclei near the impurity and an indirect coupling, through spin diffusion, to far nuclei. ${ }^{15}$ Spin diffusion is the process by which information about the lattice temperature is carried to these remote nuclear spins through a series of mutual flips between neighboring spins. For the slow diffusion case, the diffusion process is slow enough that the paramagnetic center can give up energy to the lattice faster than the energy can diffuse to this ion from the nuclei. Conversely, in the rapid diffusion case, the diffusion is fast enough that the energy from the nuclear spins diffuses to the paramagnetic center faster than the center can give up the energy to the lattice. Also, in this latter case the spins are almost all at the same spin temperature, which they are not for the slow diffusion case.

Before proceeding further with the discussion of paramagnetic impurity relaxation, there are several terms which must be defined: $a=$ nearest-neighbor distance (between like nuclei), $T_{2}=$ nuclear-spin-spin relaxation time, $b=$ spin-diffusion barrier radius, $D=$ diffusion constant $\left(\simeq a^{2} / 50 T_{2}\right), N_{I}=$ concentration of paramagnetic impurities, $R=$ average distance between impurities, $C / r^{6}=$ transition probability of direct flip of nuclear spin at distance $r$ from impurity [see Eq. (5)], $\beta=(C / D)^{1 / 4}, \delta=\frac{1}{2}(\beta / b)^{2}$.

The spin-diffusion barrier radius $b$ is so called because inside $b$ the magnetic field gradient is too large to permit spin diffusion. (The gradient arises because the dipolar interaction with the paramagnetic center varies with distance from the center.) Note that this $b$ is not necessarily the same as $b_{0}$, the radius inside of which the nuclei are shifted out to the wings of the resonance line. Blumberg ${ }^{15}$ does not differentiate between the two and when $\tau_{c}$ (the electron correlation time) is very much less than $T_{2}$, he gives $b \propto(H / T)^{1 / 3}$. Lowe and Tse, ${ }^{16}$ for the same restrictions on $\tau_{c}$, give $b \propto(H / T)^{1 / 4}$ and $b_{0} \propto$ $(H / T)^{1 / 3}$. (The $b$ of Blumberg is the $b_{0}$ of Lowe and Tse).

Jerome $e t a l .^{17}$ have proposed that the magnetization gradient, and therefore a $b$, can arise from a scalar-contact interaction with the partially localized electrons. They cite Blumberg's formulation of $b$ but use a $\left\langle\mu_{z}\right\rangle$ appropriate to the electron gas around the impurity ion rather than to the magnetic moment of the ion itself. To calculate $b$ for this case, the spatial dependence of the electronic wave functions must be known.

For the case of a powder where an average over the angle between the magnetic field and $\mathbf{r}$ has been taken, 
$C$ is given by ${ }^{15}$

$$
C=(1 / 5 \pi)\left(\gamma_{p} \gamma_{n} \hbar\right)^{2} S(S+1)\left[\tau_{c} /\left(1+\left(\omega \tau_{c}\right)^{2}\right)\right],
$$

where $\gamma_{p}$ is the gyromagnetic ratio of the paramagnetic center, $S$ is the spin of the center, and $\omega$ is the resonance frequency. For $\mathrm{CdO}$, the value of $\omega \tau_{c}$ is $\ll 1$.

For rapid diffusion to occur, there are two relationships which must be true. ${ }^{16}$ The first is that $\delta$ be very much less than 1 or, equivalently, $b \gg \beta$, and the second requirement is that $R$ be greater than $b$. If $R \gg \beta \gg b$, then the diffusion-limited case is applicable. In $\mathrm{CdO}$ we are concerned with the rapid diffusion case of paramagnetic impurity relaxation where $T_{1}$ is given by ${ }^{15}$

$$
1 / T_{1}=\frac{4}{3} \pi N_{I} C b^{-3} \text {. }
$$

Depending on which expression for $b$ we use, $T_{1}$ is proportional to $(H / T)^{x}$, where $x=0.75$ (Lowe and Tse $\left.^{16}\right)$ or $x=1.0$ (Blumberg ${ }^{15}$ ).

\section{Knight Shift}

The Knight shift occurs primarily in metals and is caused by the polarization of the Fermi-level electrons by the magnetic field. Zhogolov has suggested that there is also a Knight shift in semiconductors; however, he estimated that it was several orders of magnitude smaller than in a metal. ${ }^{18}$ Assuming Pauli susceptibility, we can use the scalar-contact term of the Hamiltonian [Eq. (1)] to calculate the Knight shift $(K)$ for degenerate electrons ${ }^{13}$ :

$$
\Delta H / H \equiv K=-(8 / 9 \pi)^{1 / 3} \gamma_{e}^{2} m_{e}{ }^{*} V\left\langle\left|\phi_{F}(0)\right|^{2}\right\rangle N_{e}^{1 / 3} .
$$

Equation (7) predicts that, at least for metal, $K$ should be independent of $H$ and $T$, neglecting higher-order effects, and should vary as $N_{e}^{1 / 3}$. The value of $K$ would be expected to decrease rapidly as the Fermi level falls below the conduction band because the electronic density at nuclei in the bulk should decrease. ${ }^{11}$

Another useful parameter is the Korringa product, $T_{1} T K^{2}$ which, for $\mathrm{CdO}$, we get from Eqs. (4) and (7) as

$$
T_{1} T K^{2}=\gamma_{e}^{2} \hbar / \gamma_{n}^{2} 4 \pi k=5.36 \times 10^{-6} .
$$

The experimental values of the Korringa product should be constant as a function of $T$ and $N_{e}$ for a metal and therefore this product can be a sensitive test of whether a compound is metallic.

\section{EXPERIMENTAL PROCEDURE AND RESULTS}

\section{Sample Preparation}

There were two different types of $\mathrm{CdO}$ samples used in the course of this investigation. The first was a collection of $\mathrm{CdO}$ polycrystals grown by the vapor-phase deposition process with a vaporization temperature of about $1475^{\circ} \mathrm{K}$ and a condensation temperature of $1275^{\circ} \mathrm{K}$. The resultant sample was designated as CdO 5 and has been studied before. ${ }^{9}$ Two variations were made
TABLE I. Carrier concentration of CdO samples (measured at $\left.4.2^{\circ} \mathrm{K}\right) .^{\mathrm{a}}$

\begin{tabular}{ll}
\hline \hline Sample & $\begin{array}{c}\text { Concentration } N_{e} \\
\left(\mathrm{~cm}^{-3}\right)\end{array}$ \\
\hline CdO 10 & $4.4 \times 10^{19}$ \\
CdO 8 & $2.4 \times 10^{19}$ \\
CdO 5 & $1.9 \times 10^{19}$ \\
CdO 6A & $7.4 \times 10^{18}$ \\
CdO 2A & $3.7 \times 10^{18}$ \\
CdO 9A & $3.3 \times 10^{18}$ \\
\hline
\end{tabular}

${ }^{\mathrm{a}} N_{e}$ for CdO 10 was averaged over temperature because of large experimental uncertainties (see text).

of CdO 5: (1) by doping it with indium to give sample $\mathrm{CdO} 10$; and (2) by sintering $\mathrm{CdO} 5$ in air at $800^{\circ} \mathrm{K}$ for $5 \mathrm{~h}$ to get the sample designated $\mathrm{CdO} 6 \mathrm{~A}$. The sintering temperature of $800^{\circ} \mathrm{K}$ was chosen as a result of the work of Jarzebski, ${ }^{19}$ who reported that $800^{\circ} \mathrm{K}$ seemed to give the lowest concentration.

The second type of $\mathrm{CdO}$ sample was a powder, composed of small crystals as verified by x-ray diffraction experiments. There were three samples made from this powder: (1) $\mathrm{CdO} 8$ was just the plain powder; (2) CdO $2 \mathrm{~A}$ was $\mathrm{CdO} 8$ sintered for $5 \mathrm{~h}$ at $800^{\circ} \mathrm{K}$ in air; (3) $\mathrm{CdO} 9 \mathrm{~A}$ was $\mathrm{CdO} 8$ sintered for $16 \mathrm{~h}$ at $800^{\circ} \mathrm{K}$ with oxygen flowing over the powder. Other sintering times, temperatures, and oxygen pressures (up to about $50 \mathrm{~atm}$ ) were tried but none of these lowered the carrier concentration below $3.3 \times 10^{18} \mathrm{~cm}^{-3}$. The carrier concentrations of all six CdO samples, as determined by Hall measurements at $4.2^{\circ} \mathrm{K}$, are shown in Table I.

\section{NMR Measurements}

A Varian V-4200B wide-line NMR spectrometer equipped with the variable temperature accessory (nitrogen gas flow) was used to perform the relaxationtime and Knight-shift measurements from 90 to $300^{\circ} \mathrm{K}$. These temperatures were measured by a copperconstantan thermocouple. A two-chamber Pyrex Dewar, to which the crossed-coil probe could be clamped, was used with liquids nitrogen and helium to achieve the lower temperatures. All data were taken at temperatures below the Fermi temperature $\left(T_{F} \simeq 400^{\circ} \mathrm{K}\right.$ for $N \simeq 3 \times 10^{18} \mathrm{~cm}^{-3}$ and $m_{e}{ }^{*} \simeq 0.2 m_{e}$ ).

The $T_{1}$ measurements were made using the direct saturation-recovery technique. The $\mathrm{Cd}^{113}$ spins were saturated at the resonance field $H_{0}$ with a high rf field. The main field was then quickly moved about $10 \mathrm{G}$ from $H_{0}$, while the rf field was maintained at full strength. The spin system was allowed to partially recover and then the magnetic field was swept through resonance to observe a fast passage dispersion signal. The signal height was proportional to the magnetization that had recovered during the off-resonance time period. This procedure, which could be performed 


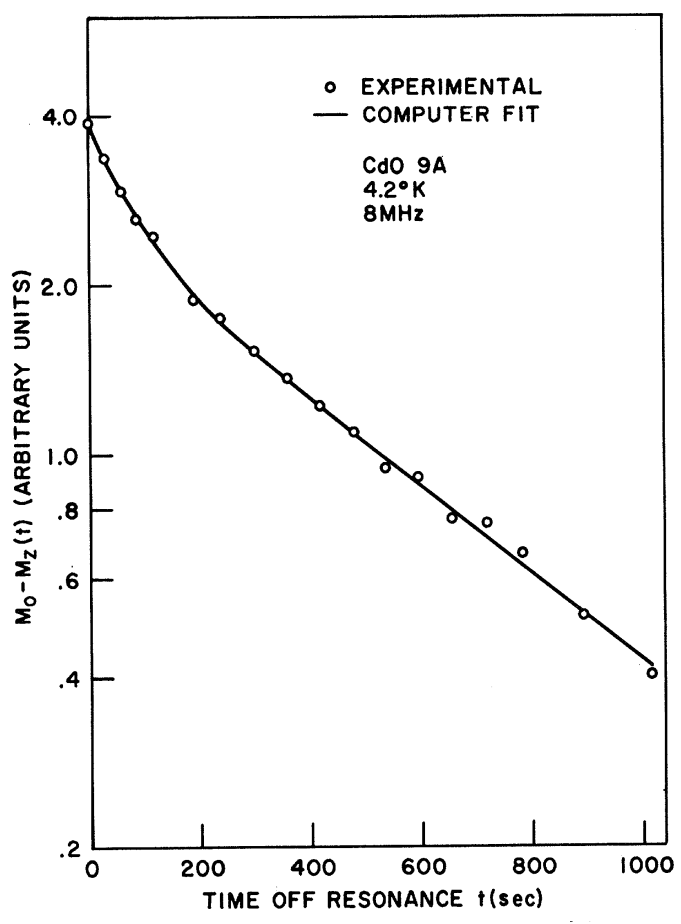

FIG. 1. Semilog plot of $\left[M_{0}-M_{z}(t)\right]$ versus $t$ showing curvature of magnetization recovery.

manually or electronically, was repeated for several off-times. The $z$ component of the magnetization recovered in accordance with the following equation:

$$
M_{z}(t)=M_{0}\left(1-e^{-t / T_{1}}\right),
$$

where $M_{0}$ is the equilibrium value of $M_{z}(t)$. The value of $M_{0}$ was determined by leaving the field off resonance for at least four $T_{1}$ 's. A semilog plot of $M_{0}-M_{z}(t)$ versus $t$ was made and, as expected, the curve was a straight line with slope $1 / T_{1}$, except for some samples, as explained below.

At temperatures of $4.2^{\circ} \mathrm{K}$ and below and for $N_{e}$ equal to or less than $2.4 \times 10^{19} \mathrm{~cm}^{-3}$, the curve of $\ln \left[M_{0}-\right.$ $\left.M_{z}(t)\right]$ versus $t$ was not a straight line. A typical example is shown in Fig. 1. For all measurements yielding a curved line the data could be fitted to the sum of two exponentials in the following form:

$$
M_{0}-M_{z}(t)=\left[M_{0} /(1+\alpha)\right]\left(\alpha e^{-t / T_{11}}+e^{-t / T_{12}}\right),
$$

where $T_{11}$ and $T_{12}$ are two independent relaxation times and $\alpha$ is a constant for a given curve. We designated $T_{12}$ as the longer of the two $T_{1}$ 's and calculated its value from the slope of the asymptotic part of the curve (slope $=-1 / T_{12}$ ) and then used a computer to iterate $T_{11}$ and $\alpha$ to get the best over-all fit to the curve. Although the fits were generally good, it is quite possible that the calculated $T_{11}$ is actually an average value, i.e., there could well be a small spread of $T_{11}$ values.

The results of the nuclear-spin-lattice relaxation- time measurements for $\mathrm{CdO} 10,8,6 \mathrm{~A}$, and $9 \mathrm{~A}$ are plotted versus temperature at $8 \mathrm{MHz}$ in Figs. 2-5, respectively. For $\mathrm{CdO} 10$, no curvature was seen and $T_{1} T=81 \pm 6 \mathrm{sec}^{\circ} \mathrm{K}$ independent of temperature $(1.4$ $\left.300^{\circ} \mathrm{K}\right)$ and frequency $(2-8 \mathrm{MHz})$.

For $\mathrm{CdO} 8, T_{1} T=111 \pm 8 \mathrm{sec}^{\circ} \mathrm{K}$ from 77 to $370^{\circ} \mathrm{K}$ and $T_{1}$ was independent of frequency $(2-10 \mathrm{MHz})$. There was curvature at $4.2^{\circ} \mathrm{K}$ and below; here $T_{12} T=$ $200 \pm 20 \sec ^{\circ} \mathrm{K}$ and was independent of temperature and frequency. The temperature variation of $T_{11}$ was $T^{-0.9 \pm 0.1}\left(\leq 4.2^{\circ} \mathrm{K}\right)$. Also, $T_{11}$ was slightly frequency dependent, decreasing by $25 \%$ from 10 to $2 \mathrm{MHz}$. Since the accuracy of $T_{11}$ is not much better than $\pm 20 \%$, no specific power dependence of $T_{11}$ on frequency could be determined.

The sample $\mathrm{CdO} 6 \mathrm{~A}$ had $T_{1} \propto T^{-0.9}$ for 77 to $300^{\circ} \mathrm{K}$; the $T_{1}$ appeared independent of frequency $(2-8 \mathrm{MHz})$ and no curvature was seen. Both $T_{11}$ and $T_{12}$ were proportional to $T^{-1}\left(1.4-4.2^{\circ} \mathrm{K}\right)$. No detailed investigation of the frequency dependence of $T_{11}$ and $T_{12}$ was undertaken because if there was a dependence, it was on the order of the experimental error. (The filling factor for this sample was low and so the $S / N$ was good only at $8 \mathrm{MHz}$, the best frequency for the probe.)

The nuclear-spin-lattice relaxation time of sample CdO 9A varied roughly as $T^{-1 / 2}$ from 300 to $120^{\circ} \mathrm{K}$ and was independent of frequency $(2-10 \mathrm{MHz})$. As the temperature was decreased below $120^{\circ} \mathrm{K}$, the $T_{1}$ increased much more slowly than $T^{-1 / 2}$. For $\mathrm{CdO} 9 \mathrm{~A}$,

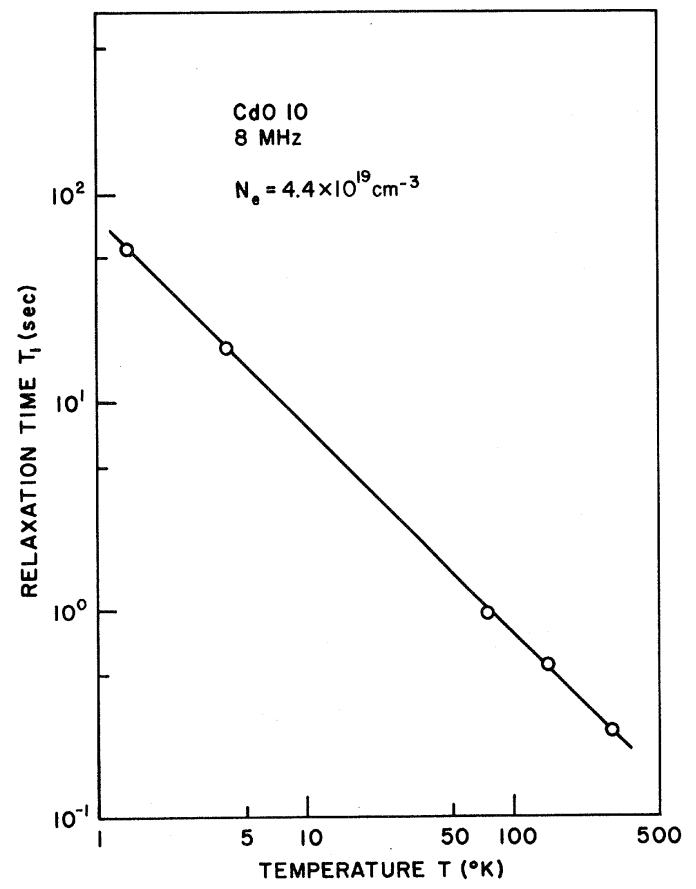

FIG. 2. Nuclear-spin-lattice relaxation time as a function of temperature for $\mathrm{CdO} 10$ at $8 \mathrm{MHz}$. The slope of the line is $T^{-1}$. 
curvature of $\ln \left[M_{0}-M_{z}(t)\right]$ versus $t$ first appeared at $77^{\circ} \mathrm{K}$. For temperatures of $4.2^{\circ} \mathrm{K}$ and below, $T_{12}$ varied as $T^{-1}$, as did $T_{11}$. The frequency dependence of $T_{11}$ and $T_{12}$ are shown in Fig. 6, where $T_{11} \propto \nu^{0.3}$ and $T_{12} \propto \nu^{0.6}$ at $4.2^{\circ} \mathrm{K}$.

The second type of NMR experiments were the resonance field shift measurements in $\mathrm{CdO}$. The measurements were performed using the same wide-line spectrometer. The dispersion mode was used and the field sweep width was $10 \mathrm{G}$. The main field was swept slowly both up and down field through resonance to minimize any possible drift in the electronics. The resonance field for each of the $\mathrm{CdO}$ samples was compared with that of a water solution of $\mathrm{CdCl}_{2}$, which was used as an unshifted standard. The errors in the measurements are estimated to be about $\pm 0.2 \mathrm{G}$ for each resonance field, so that the maximum error in $\Delta H$ is $\pm 0.4 \mathrm{G}$. As will be seen, this $0.4 \mathrm{G}$ is sometimes a large fraction of the size of $\Delta H$. The resonance shifts were measured at 300 and $4.2^{\circ} \mathrm{K}$.

The resonance shift in $\mathrm{CdO}$ has two paramagnetic contributions, the Knight shift, described earlier, and a chemical shift caused by interaction of the nuclear spins with valence-electron orbital magnetic moments polarized by the magnetic field. ${ }^{12,13}$ Cadmium oxide is estimated to have about $20 \%$ covalent bonding. ${ }^{20}$ Other Cd compounds, such as CdTe, CdSe, and CdS, which are more covalent than $\mathrm{CdO}$, have resonance shifts of 0.013 to $0.051 \%$, respectively, ${ }^{9}$ and these shifts were judged to be almost entirely due to chemical shifts because the carrier concentrations were too low

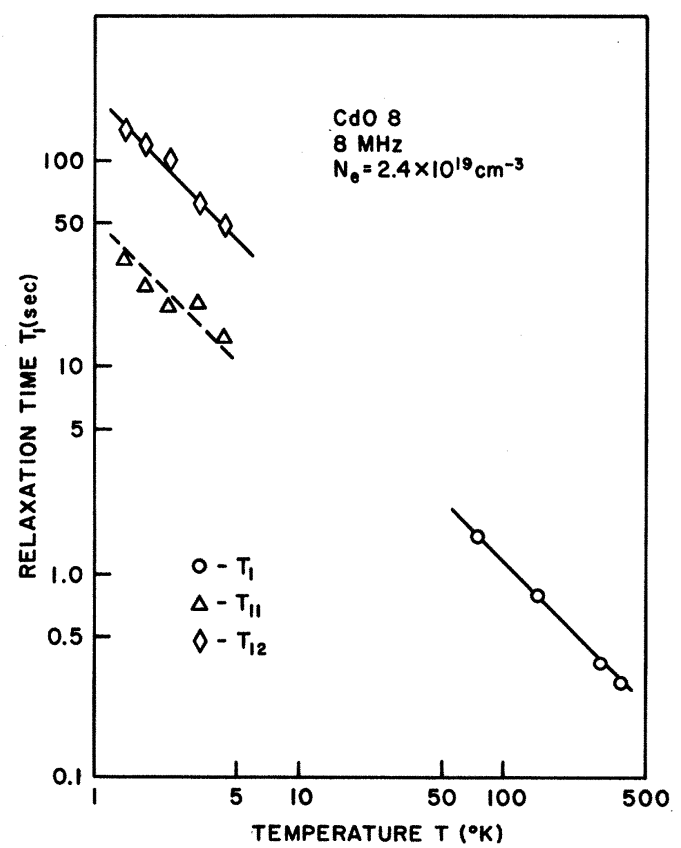

FIG. 3. Nuclear-spin-lattice relaxation time as a function of temperature for CdO 8 at $8 \mathrm{MHz}$. The slope of all the lines is $T^{-1}$.

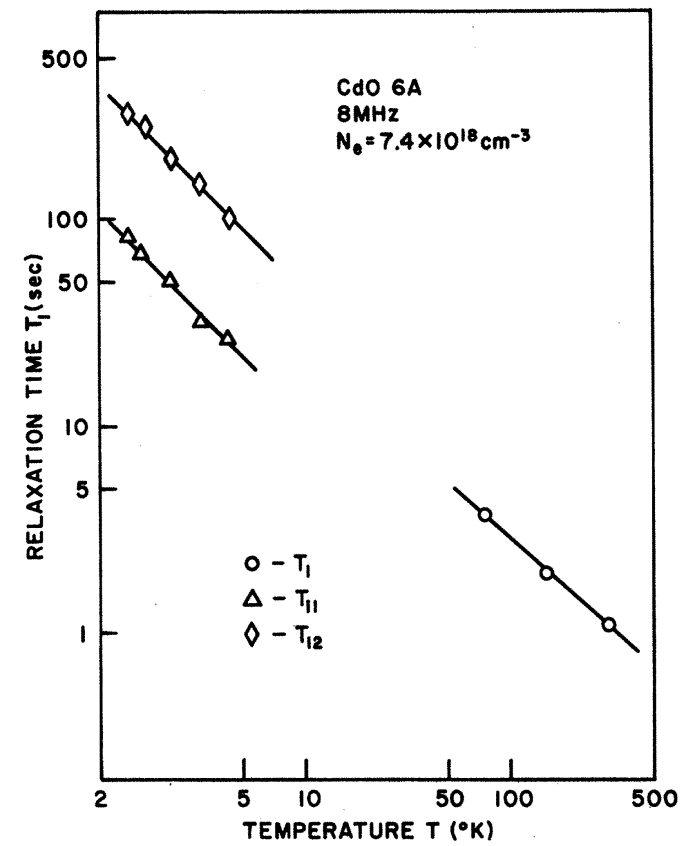

FIG. 4. Nuclear-spin-lattice relaxation time as a function of temperature for $\mathrm{CdO} 6 \mathrm{~A}$ at $8 \mathrm{MHz}$. The slope of the $T_{11}$ and $T_{12}$ lines is $T^{-1}$ and the slope of the $T_{1}$ line is $T^{-0.9}$.

for the Knight shift to be significant. By comparison, the size of the chemical shift in $\mathrm{CdO}$ was estimated to be less than $0.013 \%$.

The theoretical Knight shift for $\mathrm{CdO} 10$ was calculated from the Korringa product [Eq. (8)] and was found to be $0.027 \%$ or down by $0.011 \%$ from the experimental value of $0.038 \%$. From this we believe that the $0.011 \%$ is about the size of the chemical shift in $\mathrm{CdO}$, consistent with the aforementioned estimate. This conclusion is substantiated by the fact that when the $0.011 \%$ was subtracted from the experimental shifts of $\mathrm{CdO} 8$ and 5 , the remaining shift was just that which was calculated from the Korringa product. The value of $0.011 \%$ was thus subtracted from all the resonance shifts to give the Knight shifts which are plotted in Fig. 7 at $300^{\circ} \mathrm{K}$ and $4.2^{\circ} \mathrm{K}$ as a function of carrier concentration. At high $N_{e}$, the Knight shift varies as $N_{e}^{1 / 3}$ and is independent of temperature. For the three CdO samples with the lowest $N_{e}, K$ decreases with temperature.

\section{Hall Measurements}

Each of the $\mathrm{CdO}$ powder samples was pressed into a cylinder under about 1000 -atm pressure. These cylinders were then cut into rectangular parallelepipeds with cross sections of about $5 \times 5 \mathrm{~mm}^{2}$ and lengths of about $7 \mathrm{~mm}$. Ohmic contacts were made using In solder and an ultrasonic soldering iron. A five-lead configuration ${ }^{21}$ was employed to minimize contact resistance problems. 
TABLE II. Experimental measurements on CdO.

\begin{tabular}{ccccc}
\hline \hline Sample No. & $\begin{array}{c}\text { Temperature } \\
T\left({ }^{\circ} \mathrm{K}\right)\end{array}$ & $\begin{array}{c}\text { Resistivity } \\
\rho(\Omega \mathrm{cm})\end{array}$ & $\begin{array}{c}\text { Hall mobility } \\
\mu_{H}\left(\mathrm{~cm}^{2} / \mathrm{V} \mathrm{sec}\right)\end{array}$ & $\begin{array}{c}\text { Concentration } \\
N_{e}\left(10^{19} \mathrm{~cm}^{-3}\right)\end{array}$ \\
\hline \multirow{2}{*}{10} & 300.0 & 0.099 & 1.4 & 4.46 \\
& 77.0 & 0.139 & 1.1 & 4.00 \\
& 4.2 & 0.170 & 0.7 & 5.00 \\
& 300.0 & 0.218 & 1.2 & 2.45 \\
& 47.0 & 0.216 & 1.3 & 2.30 \\
& 4.2 & 0.222 & 1.2 & 2.43 \\
& 300.0 & 0.069 & 5.2 & 1.8 \\
& 77.0 & 0.086 & 4.0 & 1.8 \\
& 4.2 & 0.095 & 3.5 & 1.9 \\
& 300.0 & 0.110 & 9.2 & 6.3 \\
& 77.0 & 0.098 & 8.5 & 7.4 \\
& 4.2 & 0.133 & 6.3 & 7.4 \\
& 300.0 & 0.031 & 72 & 2.8 \\
& 77.0 & 0.024 & 98 & 2.6 \\
& 4.2 & 0.030 & 56 & 3.7 \\
\hline
\end{tabular}

A magnetic field of $10 \mathrm{kG}$ and crystal currents of 100 and $200 \mathrm{~mA}$ were used to make the Hall-effect measurements.

The results of these experiments on $\mathrm{CdO} 10,8,5,6 \mathrm{~A}$ and $2 \mathrm{~A}$ at 300,77 , and $4.2^{\circ} \mathrm{K}$ are shown in Table II. For the three high $N_{e}$ samples, the carrier concentration

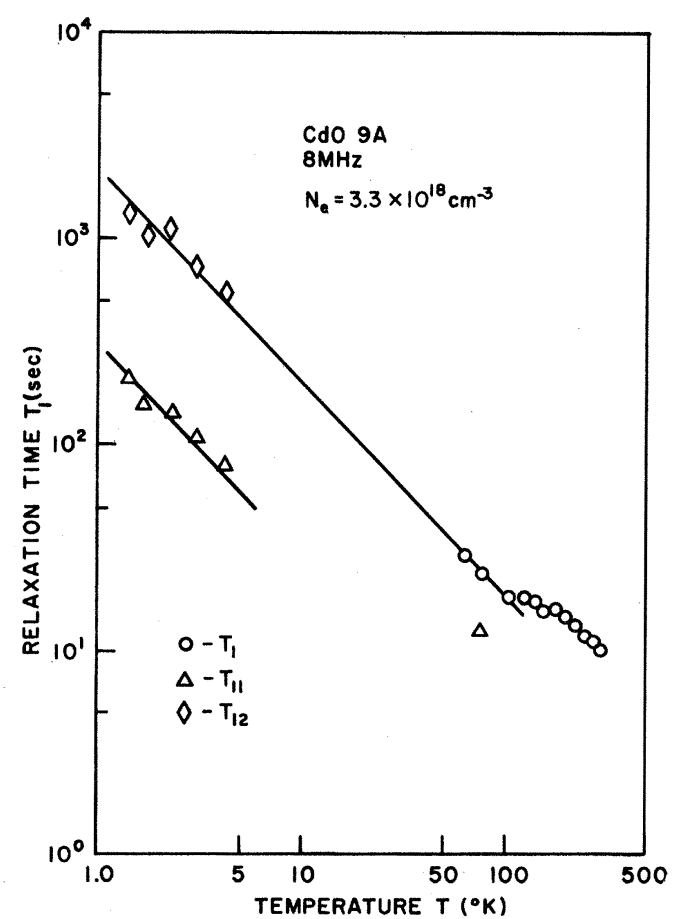

Fig. 5. Nuclear-spin-lattice relaxation time as a function of temperature for CdO $9 \mathrm{~A}$ at $8 \mathrm{MHz}$. The slope of the lines is $T^{-1}$. was independent of temperature. The Hall voltages for $\mathrm{CdO} 6 \mathrm{~A}$ and $2 \mathrm{~A}$ were observed to have a hump as the samples warmed up to room temperature from $4.2^{\circ} \mathrm{K}$. The precision of these data is about $5 \%$ whereas the accuracy is probably near $20 \%$ due to such things as

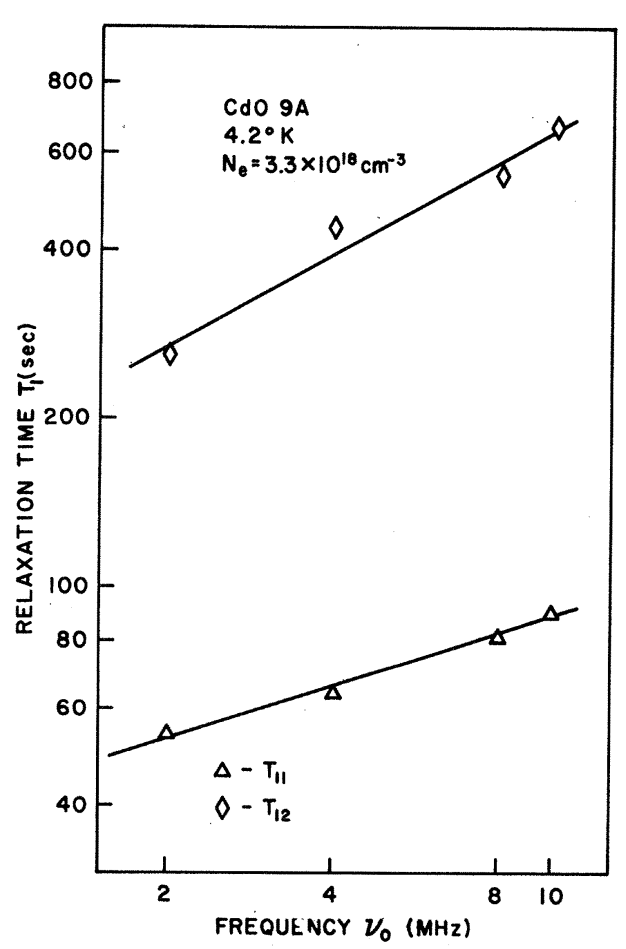

FIG, 6. Nuclear-spin-lattice relaxation time as a function of frequency for $\mathrm{CdO} 9 \mathrm{~A}$ at $4.2^{\circ} \mathrm{K}$. The slope of the two lines obtained by least-squares fit are $\nu^{0.6}$ for $T_{12}$ and $\nu^{0.3}$ for $T_{11}$. 
finite size contacts (rather than point contacts) and variations in crystalline cross section. The larger excursion in $N_{e}$ for CdO 10 was because the Hall voltage was $2.0 \pm 0.5 \mu \mathrm{V}$, which was a $25 \%$ uncertainty. The other two high $N_{e}$ samples (8 and 5) had much higher Hall voltages $(\sim 10 \mu \mathrm{V})$, giving only the $5 \%$ uncertainty. The Hall voltage did not reverse upon field reversal at $4.2^{\circ} \mathrm{K}$, indicating magnetoresistivity, discussed below.

A more detailed investigation was made of $\mathrm{CdO} 9 \mathrm{~A}$ and the behavior of $\rho, \mu_{H}$, and $R_{H}(=1 / N e c)$ are shown in Fig. 8. There is a very definite hump in the Hall constant centered at about $120^{\circ} \mathrm{K}$. The mobility could be fitted to the following equation in the temperature range $42-300^{\circ} \mathrm{K}$ :

$$
1 / \mu_{H}=1.0 \times 10^{-3} T^{+1 / 2}+4.8 T^{-3 / 2} .
$$

Below $42^{\circ} \mathrm{K}, \mu_{H}$ was approximately constant. At high temperature $\left(T>120^{\circ} \mathrm{K}\right)$, the mobility is dominated by optical phonon scattering 22 for which $\mu_{H} \propto T^{-1 / 2}$. This relationship is true only for nondegenerate electrons in ionic solids. For $T$ less than $120^{\circ} \mathrm{K}$, the mobility of $\mathrm{CdO} 9 \mathrm{~A}$ is governed by ionized impurity scattering 22 and $\mu_{H} \propto T^{3 / 2}$. At very low temperatures, $\rho$ and $\mu_{H}$ are constant, implying that the electrons are degenerate.

Finally, it should be mentioned that some preliminary magnetoresistance measurements were made on a small crystal, similar to $\mathrm{CdO} 5$, and on $\mathrm{CdO} 9 \mathrm{~A}$. At $4.2^{\circ} \mathrm{K}$, from $H=1$ to $11 \mathrm{kG}$, the magnetoresistance was negative for both samples but the power dependence of $\Delta \rho / \rho$ on $H$ was lower for the high-concentration sample (5) than for the low-concentration one (9A). This is consistent with the findings of Khosla and Fischer ${ }^{23}$ for degenerate chlorine-doped CdS. Further studies must be made before any conclusions are drawn about the mechanism leading to this phenomenon.

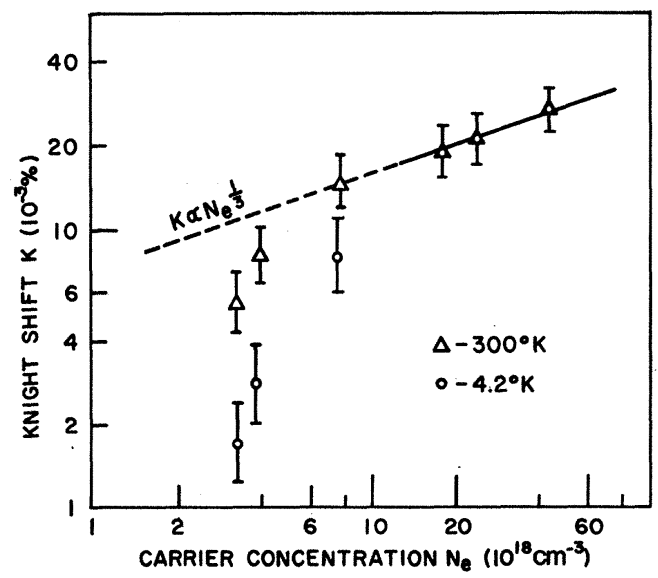

FIG. 7. Dependence of the Knight shift in $\mathrm{CdO}$ as a function of carrier concentration shown at 300 and $4.2^{\circ} \mathrm{K}(8 \mathrm{MHz})$. Note that the three highest concentration points represent both temperatures.

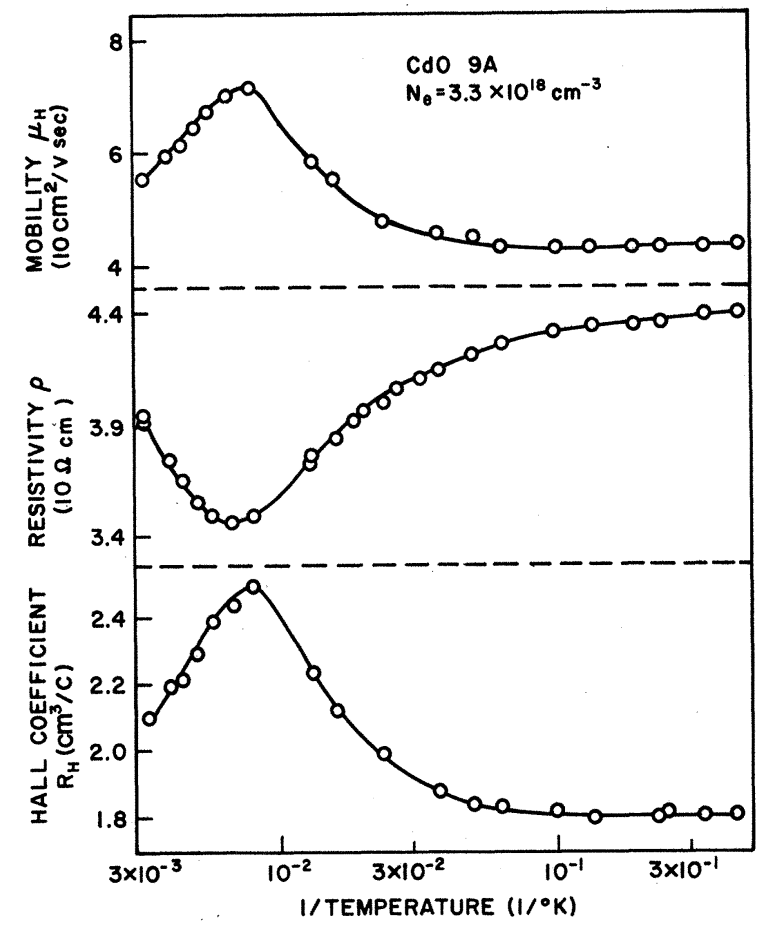

FIG. 8. Hall-effect measurements of $\mu_{H}, \rho$, and $R_{H}$ as a function of temperature in $\mathrm{CdO} 9 \mathrm{~A}$.

\section{Discussion}

Figure 9 shows the variation of $T_{1}$ in $\mathrm{CdO}$ with $N_{\epsilon}$. As can be seen for the four high-carrier-concentration samples (CdO 10, 8, 5, and 6A), $T_{1}$ varies as $N_{e}^{-2 / 3}$. In addition, as was shown in Sec. III, the $T_{1}$ 's of CdO 10,8 , and 5 varied as $T^{-1}$, at least from $77^{\circ}$ to $300^{\circ} \mathrm{K}$. These relationships indicate that the $\mathrm{Cd}^{113}$ nuclei in these $\mathrm{CdO}$ powders are being relaxed by degenerate electrons [see Eq. (4)]. In order to calculate $\left\langle\left|\phi_{F}(0)\right|^{2}\right\rangle$ from Eq. (4), we needed to choose a value for $m_{e}{ }^{*}$. Values of $m_{e}{ }^{*}$ in the literature vary from $0.1 m_{e}$ to $0.3 m_{e},{ }^{24}$ depending on the type of measurements that have been made. Miloslavskii and Ranyuk ${ }^{25}$ have determined that $m_{e}{ }^{*} \simeq 0.2 m_{e}$ at the bottom of the conduction band, which was in good agreement with the $m_{e}{ }^{*}$ obtained by Look $^{9}$ from a fit of $\mathrm{CdO}$ mobility to the Conwell-Weisskopf mobility relation. Therefore, we believe that for $\mathrm{CdO} m_{e}{ }^{*}=0.2 m_{e}$, within about $20 \%$.

Using a value of $0.2 m_{e}$ for $m_{e}{ }^{*}$ we calculate, from Eq. (4), $V\left\langle\left|\phi_{F}(0)\right|^{2}\right\rangle \simeq 2200$, where the wave function is normalized to unity in a unit volume. Normalizing to unity in an atomic volume gives $\left\langle\left|\phi_{F}(0)\right|^{2}\right\rangle \equiv P_{F}=$ $8.5 \times 10^{25} \mathrm{~cm}^{-3}$. This is the relative density at the nucleus compared to the average value throughout the crystal. The relative electronic probability density for the free $\mathrm{Cd}$ ion is $\left\langle\left|\psi_{A}(0)\right|^{2}\right\rangle \equiv P_{A}=9.2 \times 10^{25} \mathrm{~cm}^{-3}$ using the Goudsmit formula for the $4 d^{10} 5 s^{2} S_{1 / 2}$ term. ${ }^{9}$ This gives the ratio $\xi=P_{F} / P_{A}$ as 0.92 , which is in the 


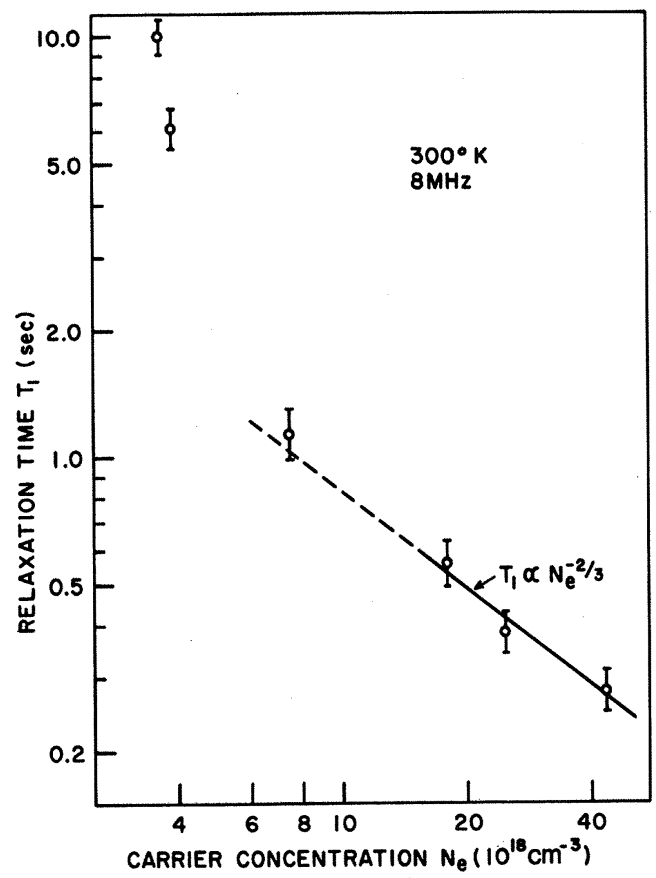

FIG. 9. Nuclear-spin-lattice relaxation time as a function of carrier concentration in $\mathrm{CdO}$ at $300^{\circ} \mathrm{K}$ and $8 \mathrm{MHz}$.

range typical for metals. ${ }^{13}$ The size of this ratio indicates that the electrons on the Fermi surface are characteristic of the host lattice conduction band and, therefore, the Fermi level is in the conduction band. If the electrons were confined to the regions near impurity centers, $\xi$ would be expected to be smaller because of the smaller interaction with the nuclei in the bulk. We thus conclude that electrons are degenerate and in the conduction band from 1.4 to $300^{\circ} \mathrm{K}$ for $\mathrm{CdO} 10$, and from 77 to $300^{\circ} \mathrm{K}$ for $\mathrm{CdO} 8$ and 5 . The two $T_{1}$ 's seen in $\mathrm{CdO} 8$ and 5 at $4.2^{\circ} \mathrm{K}$ and below will be discussed later in this section.

The Knight-shift data on $\mathrm{CdO} 10,8$, and 5 further support the belief that the scalar contact hyperfine interaction is the dominant one in these samples from the fact that $K$ varies as $N_{e}^{1 / 3}$ and is independent of temperature. Also, the carrier concentration $\left(\propto R_{H}{ }^{-1}\right)$ is independent of temperature from 4.2 to $300^{\circ} \mathrm{K}$. The presence of a hump in $R_{H}$ versus $T$ generally is taken to indicate that the impurity band is separate from the conduction band ${ }^{7}$; however, such a hump was not seen. All of these data lead us to conclude that in $\mathrm{CdO}$ with $N_{e}$ greater than $10^{19} \mathrm{~cm}^{-3}$, the impurity band (or the Fermi level) overlaps the conduction band and the electrons are degenerate over the temperature range of our experiment.

The relaxation time of $\mathrm{CdO} 9 \mathrm{~A}$ is apparently not caused by degenerate conduction-band electrons as $T_{1} T$ is not a constant (see Fig. 5) in the region $77-300^{\circ} \mathrm{K}$, nor does $T_{1}$ vary as $N_{e}^{-2 / 3}$ (see Fig. 9). However, in the temperature range $120-300^{\circ} \mathrm{K}$, the product $T_{1} N_{e} T^{1 / 2} \simeq$ $(5.1 \pm 0.5) \times 10^{20} \sec ^{\circ} \mathrm{K}^{1 / 2} \mathrm{~cm}^{-3}$ and the $T_{1}$ 's are independent of frequency $(2-10 \mathrm{MHz})$. This is the relationship that would be expected if the relaxation agent were nondegenerate conduction electrons. Using $m_{e}{ }^{*}=$ $0.2 m_{e}$ and the equation for nondegenerate electron relaxation ${ }^{13}$ to calculate the value of the electronic probability density, we can arrive at a value of $\xi=0.39$ for CdO 9A, lower than expected.

As the temperature was decreased further from 120 to about $77^{\circ} \mathrm{K}$, the $T_{1}$ did not change very much. At $77^{\circ} \mathrm{K}$, the curvature of $\ln \left[M_{0}-M_{z}(t)\right]$ versus $t$ first appeared and the long $T_{1}$ component $\left(T_{12}\right)$ was frequency dependent. At $8 \mathrm{MHz}, T_{11}=16 \mathrm{sec}, T_{12}=35 \mathrm{sec}$, and at $2 \mathrm{MHz}, T_{12}=25 \mathrm{sec}$. In this temperature range, it appears that the electrons are localizing near impurity centers. No specific temperature dependence could be established here because of the inability to separate two $T_{1}$ 's.

For all the $T_{1}$ data taken on $\mathrm{CdO} 9 \mathrm{~A}$ at $T \leq 4.2^{\circ} \mathrm{K}$, curvature was present, giving rise to the two $T_{1}$ 's shown in Figs. 5 and 6. The curvature is not due to quadrupolar effects since $\mathrm{Cd}^{113}$ has spin $\frac{1}{2}$. It is also unlikely that the curvature is mainly due to the powder nature of $\mathrm{CdO}$ since it was not seen at all temperatures or in all samples. Furthermore, slow nuclear-spin diffusion, which can also cause curvature, is not applicable in our samples. We believe the origin is two different spin systems that are relaxed independently. The magnetization of each system recovers in accordance with Eq. (9) but the signal we measure is really the sum of the magnetization signals from the two sets of spins. If one associates a different $T_{1}$ with each, and then sums two equations like Eq. (9), we can obtain Eq. (10), where now $M_{0}$ is the sum of the equilibrium values of each system. The origins of the $T_{1}$ 's will be discussed in the next section.

As shown in Fig. 7, the Knight shifts of $\mathrm{CdO} 6 \mathrm{~A}$, $2 \mathrm{~A}$, and $9 \mathrm{~A}$ decrease with decreasing temperature. Again this result occurs because the electronic probability density in the bulk decreases as the electrons become more localized near impurity centers. As expected, the experimental Korringa product (see Table III) is lower than the theoretical value and appears to be decreasing as the carrier concentration is lowered.

TABLE III. Korringa products for cadmium oxide at $4.2^{\circ} \mathrm{K}$.

\begin{tabular}{|c|c|c|c|}
\hline Sample No. & $T_{1}(\mathrm{sec})$ & $K\left(10^{-4} \%\right)$ & $\begin{array}{c}T_{1} T K^{2}\left(5.4 \times 10^{-6}\right. \\
\left.\sec ^{\circ} \mathrm{K}\right)\end{array}$ \\
\hline 10 & 19.9 & 2.66 & 1.0 \\
\hline 8 & 29.0 & 1.95 & 0.87 \\
\hline 5 & 38.0 & 1.78 & 0.95 \\
\hline $6 \mathrm{~A}$ & 100.0 & 0.70 & 0.39 \\
\hline $2 \mathrm{~A}$ & 320.0 & 0.30 & 0.20 \\
\hline $9 \mathrm{~A}$ & 560.0 & 0.18 & 0.14 \\
\hline
\end{tabular}


The following values of $T_{1}$ are used in calculating the Korringa product: for $\mathrm{CdO} 10$, the measured $T_{1}$; for $\mathrm{CdO} 8$ and 5 , an average of $T_{11}$ and $T_{12}$; and for $\mathrm{CdO}$ $6 \mathrm{~A}, 2 \mathrm{~A}$, and $9 \mathrm{~A}, T_{12}$. The reasons for choosing these are given in the next section.

These data and the $T_{1}$ data are interpreted to show that for $\mathrm{CdO} 6 \mathrm{~A}, 2 \mathrm{~A}$, and $9 \mathrm{~A}$, the value of $N_{e}$ is less than $N_{c b}$. The electrical data, on CdO $9 \mathrm{~A}$ in particular, support this conclusion; i.e., the hump in $R_{H}$ versus $T$ likely indicates the presence of a separate impurity band. ${ }^{7}$ The slope of $\ln \rho$ versus $1 / T$ in the temperature range $40-120^{\circ} \mathrm{K}$ gives the separation energy (or activation energy) between the two bands as about $10^{-3} \mathrm{eV}$ (for CdO 9A). The mobility behaves in the manner expected for ionized impurity scattering.

Thus, all of the NMR and Hall-effect data, and in particular, the Knight-shift and Korringa product behavior, lead to the conclusion that $N_{c b} \simeq 10^{19} \mathrm{~cm}^{-3}$. The theoretical value ${ }^{8}$ of $N_{c b}$ using an $m_{e}{ }^{*}=0.2 m_{e}$ is $1.7 \times 10^{19} \mathrm{~cm}^{-3}$ or, working backwards from the experimental value of $N_{c b}$, we conclude that $m_{e}{ }^{*} \simeq 0.17 m_{e}$, which agrees fairly well with the value chosen for $m_{e}{ }^{*}$ (within the assumed accuracy of $m_{e}^{*}$ of $\pm 20 \%$ ). If we use this value for the electron effective mass, then according to the Mott criterion, we would predict that $N_{c} \simeq 2 \times 10^{18} \mathrm{~cm}^{-3}$ just below our lowest concentration sample. Perhaps a future program will be successful in lowering the concentration of impurities in $\mathrm{CdO}$ below $N_{c}$.

\section{Proposed Model}

We believe that the two spin systems discussed above may be defined as follows: (1) those spins which are relaxed by scalar contact interactions with electrons in conduction or impurity bands, and possibly also by spin-diffusion to a paramagnetic center; and (2) those spins which are relaxed only by the spin-diffusion process. The paramagnetic centers are assumed to be the donors themselves which give rise to the impurity band. It is not obvious why the electronic distribution in the impurity band should be such that the centers possess a magnetic moment, but neither is it unreasonable. For example, the existence of "local moments" in the impurity band has been postulated ${ }^{26}$ as the reason for negative magnetoresistance in heavily doped semiconductors; this phenomenon exists in $\mathrm{CdO}$ as mentioned earlier. Furthermore, at least two EPR lines have been observed in $\mathrm{CdO}^{14}$, one of which was attributed to $\mathrm{Cd}^{+}$centers at $\mathrm{Cd}^{++}$sites. (It is possible that the donor center could be $\mathrm{Cd}^{+}$in conjunction with an oxygen vacancy.)

If the electrons are in an impurity band, i.e., localized to some extent near the donor centers, then some nuclei will be in the region of localization while others will not. Those in this region will experience a contact hyperfine interaction and will be observed in the resonance signal as long as they are not within a radius $b$ of the center. A complication is introduced by the non- uniform distribution of the centers; for example, a sample which was quasimetallic on the average could have regions in the semiconducting range and other regions in the metallic range. ${ }^{27}$ This could lead to severely inhibited spin diffusion in heavily concentrated parts of the sample, isolating those parts from other parts, and prohibiting a common spin temperature; thus, nonexponential magnetization recovery would occur, leading to two (or more) $T_{1}$ 's.

We will apply these ideas first to $\mathrm{CdO} 9 \mathrm{~A}$ and assume that $T_{12}$, the long relaxation time, is due to the rapid diffusion case of paramagnetic impurity relaxation and that these spins have no significant contact interaction. For a rough estimate of the spin-diffusion barrier radius $b$ we use a value of $\tau_{c} \simeq 4 \times 10^{-10} \mathrm{sec}$, calculated from the $\mathrm{Cd}^{+}$EPR linewidth obtained by Elschner and Schlaak, ${ }^{14}$ and $\gamma_{p}=1.57 \times 10^{7}$ and $S=\frac{1}{2}$ from the same data. Then, from Eq. (6), we calculate $b \simeq 20 \AA$. Taking $T_{2} \simeq 3.4 \times$ $10^{-4} \mathrm{sec}$ (from the 0.5-G linewidth of the $\mathrm{Cd}^{113}$ resonance line) we calculate $\beta \simeq 4.7 \AA$ so that $\delta=\frac{1}{2}(\beta / b)^{2} \ll 1$. The average distance between impurities is $R=$ $\left(3 N_{e} / 4 \pi\right)^{1 / 3} \simeq 43 \AA$, making $R \gg \beta$. These facts are consistent with our initial assumption about the origin of $T_{12}$. Furthermore, according to Eqs. (5) and (6), $T_{12}$ should vary as $(H / T)$ since $\omega \tau_{c} \ll 1$. The temperature dependence is $T^{-1}$ (see Fig. 5) and the frequency dependence is $\nu^{0.6}$, supporting the model within experimental uncertainty.

We now consider those nuclei in $\mathrm{CdO} 9 \mathrm{~A}$ which relax according to $T_{11}$, the faster time, and postulate that they are near impurity centers and experience a contact interaction. The electronic wave functions must certainly extend beyond the $b$ calculated above since the Bohr radius of a bound donor electron is already about $17 \AA$. It would be interesting to calculate the $b$ arising from the polarization of the surrounding electron gas $^{17}$ but, as mentioned previously, the spatial dependence of the wave functions must be known. The fact that $T_{11}$ varies as $T^{-1}$ and is only slightly frequency dependent supports the contact relaxation mechanism. If we use Eq. (4) with $V\left\langle\left|\phi_{F}(0)\right|^{2}\right\rangle=2200$, as for $\mathrm{CdO} 10,8$, and 5 , we calculate a $T_{11} \simeq 106 \mathrm{sec}$, about $40 \%$ higher than the measured value at $8 \mathrm{MHz}$ and $4.2^{\circ} \mathrm{K}$. There are two factors to be considered in explaining this difference. The first is that the measured $N_{e}$ is an average over the whole volume of the sample and thus undoubtedly is lower than the actual value of $N_{e}$ near a center. Secondly, the dipolar interaction with the center gives an additional contribution which, in fact, is the likely explanation for the slight frequency dependence of $T_{11}$.

The discussion for $\mathrm{CdO} 2 \mathrm{~A}$ and $6 \mathrm{~A}$ parallels that of $9 \mathrm{~A}$.

On the other end of the concentration spectrum, $\mathrm{CdO} 10$ has only a single relaxation time, attributed to the fact that almost the whole sample has $N>N_{c b}$ so that all the nuclei are dominated by the contact interaction with electrons in the conduction band. But 
samples $\mathrm{CdO} 8$ and 5 exhibit two relaxation times even though, as we have asserted, the average $N$ is greater than $N_{c b}$. The reason, we believe, is that parts of these samples have $N_{c}<N<N_{c b}$, i.e., are quasimetallic, but nevertheless almost all of the nuclei are primarily relaxed by contact interaction. We assign $T_{12}$ to those nuclei in metallic regions, relaxed by conduction-band electrons, and $T_{11}$ to nuclei in quasimetallic regions, relaxed mainly by impurity-band electrons. This assignment is supported by the fact that both $T_{11}$ and $T_{12}$ vary as $T^{-1}$ from 1.4 to $4.2^{\circ} \mathrm{K}$, but $T_{12}$ is frequency independent while $T_{11}$ is slightly frequency dependent, most likely due to the influence of paramagnetic impurities. There must also be nuclei in the quasimetallic region which experience no contact interaction at all, but evidently the fraction is small and unobservable at these concentrations.

If we assume roughly equal numbers of nuclei associated with $T_{11}$ and $T_{12}$, and calculate an average $T_{1}$ in the region $1.4-4.2^{\circ} \mathrm{K}$, it turns out that $T_{1} T$ is a constant from 1.4 to $300^{\circ} \mathrm{K}$. Furthermore, the experimental Korringa product is the same (within experimental error) as the theoretical value (see Table III). This gives validity to our earlier assertion that the average $N$ is greater than $N_{c b}$ for $\mathrm{CdO} 8$ and 5 and that the impurity band does overlap the conduction band.

\section{SUMMARY}

The value of $N_{c b}$ is estimated to be $10^{19} \mathrm{~cm}^{-3}$ for CdO. The three samples ( $\mathrm{CdO} 10,8$, and 5) with carrier concentrations greater than $N_{c b}$ exhibit metallic properties. The spin-lattice relaxation time of these samples is due to the contact term of the hyperfine interaction between the degenerate conduction electrons and the $\mathrm{Cd}^{113}$ nuclei. At low temperatures, for $\mathrm{CdO} 8$ and 5 (but not for $\mathrm{CdO} 10$ ), there is a nonexponential recovery of the magnetization attributable to the fact that parts of these powders are quasimetallic, i.e., have $N<N_{c b}$. In the high (or metallic) concentration regions, the spin-lattice relaxation time $\left(T_{12}\right)$ is mainly due to the scalar-contact interaction between the conduction electrons and the nuclei. In the low (or quasimetallic) concentration regions, the relaxation time $\left(T_{11}\right)$ is caused by the scalar-contact interaction between the electrons localized near the impurity centers and the nuclei. The electrical measurements on $\mathrm{CdO} 10,8$, and 5 show that the average carrier concentration is independent of temperature, a metallic property. The Knight shifts of these samples vary as $N_{e}^{1 / 3}$, as expected for the degenerate conduction-electron interaction. The experimental Korringa products agree with the theoretical value within $15 \%$ for all three of these samples at high and low temperatures. Conversely, the three samples (CdO 6A, 2A, and 9A) with carrier concentration below $N_{c b}$ all exhibit quasimetallic properties. At temperatures above $77^{\circ} \mathrm{K}$, roughly, the spin-lattice relaxation time is primarily caused by the conduction-electron interaction with the nuclei. At temperatures below $77^{\circ} \mathrm{K}$, the measurements indicate that there are two spin-lattice relaxation mechanisms, one associated with the nuclei near an impurity center, and the other associated with the nuclei far from the center. The charge distribution is such that the centers carry a magnetic moment. The nuclei just outside a diffusion barrier radius $b$ of the impurity center are mainly relaxed by the scalar contact interaction with the localized electrons but also partially by the dipolar interaction with the center. The nuclei far from the impurity center are relaxed by nuclear spin diffusion (rapid diffusion case). The electrical measurements indicate the existence of an impurity band that is separated from the conduction band by about $10^{-3} \mathrm{eV}$ (in $\mathrm{CdO} 9 \mathrm{~A}$ ). The observed Knight shifts in these latter three samples are much smaller than the degenerate conduction-electron interaction would predict and they are temperature dependent. At $4.2^{\circ} \mathrm{K}$, the experimental Korringa products are far below the theoretical value, further substantiating the belief that the electrons are localized in a separate impurity band.

\section{ACKNOWLEDGMENTS}

We wish to thank D. R. Locker and Dr. G. J. Freyer for helpful discussions, and D. W. Naas and D. E. Johnson for technical assistance. $\dagger$ Based on work submitted by R. P. Benedict to the Air Force Institute of Technology in partial fulfillment of the requirements for an MS degree.

* Work sponsored by The Aerospace Research Laboratories, Office of Aerospace Research, U.S. Air Force, under Contract No. F33615-67-C-1027.

1 J. A. Bastin and R. W. Wright, Proc. Phys. Soc. (London) 68A, 312 (1955).

${ }^{2}$ R. Haul and D. Just, J. Appl. Phys. 33, 487 (1962).

${ }_{3}$ R. W. Wright and J. A. Bastin, Proc. Phys. Soc. (London) 71, 109 (1958).

${ }^{4}$ F. P. Koffyberg, Phys. Letters 30A, 37 (1969).

${ }^{5}$ C. A. Hogarth, Proc. Phys. Soc. (London) 64B, 691 (1951).

${ }_{6}^{6}$ E. F. Lamb and F. C. Tomkins, Trans. Faraday Soc. 58, 1424 (1962).
7 (a) N. F. Mott and W. D. Twose, Advan. Phys. 10, 107 (1961). (b) N. F. Mott, ibid. 16, 59 (1967).

${ }^{8} \mathrm{~T}$. Matsubara and Y. Toyozawa, Progr. Theoret. Phys. (Kyoto) 26, 739 (1961)

${ }^{9}$ D. C. Look, Phys. Rev. 184, 705 (1969).

${ }^{10}$ D. Jerome, Rev. Mod. Phys. 40, 830 (1968).

11 (a) R. K. Sundfors and D. F. Holcomb, Phys. Rev. 136, 810 (1964). (b) M. N. Alexander and D. F. Holcomb, Rev. Mod. Phys. 40, 815 (1968).

${ }_{12}$ C. P. Slichter, Principles of Magnetic Resonance (Harper and Row, New York, 1963), Chaps. 4 and 5.

${ }^{13} \mathrm{~A}$. Abragam, The Principles of Nuclear Magnetism (Oxford U. P., London, 1961), Chaps. 6 and 9.

${ }_{14} \mathrm{M}$. Elschner and B. Shlaak, Phys. Letters 24A, 10 (1967).

${ }^{15}$ W. E. Blumberg, Phys. Rev. 119, 79 (1960).

${ }^{16}$ I. J. Lowe and D. Tse, Phys. Rev. 166, 279 (1968). 
${ }^{17}$ D. Jerome, Ch. Ryter, and J. M. Winter, Physics 2, 81 (1965).

${ }_{18}$ D. A. Zhogolev, Fiz. Tverd. Tela 9, 59 (1967) [Soviet Phys. Solid State 9, 42 (1967) ].

${ }_{19}$ Z. M. Jarzebski, Acta Phys. Polon. 29, 37 (1966).

${ }^{20} \mathrm{~J}$. C. Phillips and J. A. Van Vechten, Phys. Rev. Letters 22, 705 (1969).

21 E. H. Putley, The Hall Effect and Related Phenomena (Butterworths, London, 1960), p. $42 \mathrm{ff}$.

${ }^{22}$ J. M. Ziman, Electrons and Phonons (Clarendon, London, 1960), Chap. 10.
${ }^{23}$ R. P. Khosla and J. R. Fischer, Bull. Am. Phys. Soc. 15, 316 (1960).

${ }^{24} \mathrm{~A}$ tabulation and discussion of the properties of $\mathrm{CdO}$ may be found in M. Neuberger, Cadmium Oxide Data Sheet DS-149, 1966 (unpublished). Copies may be obtained from DDC, Cameron Station, Bldg. 5, 5010 Duke St., Alexandria, Va. 22314.

${ }^{25}$ V. K. Miloslavskii and A. I. Ranyuk, Opt. i Spectroskopyia 11, 289 (1961).

${ }^{26}$ Y. Toyozawa, J. Phys. Soc. Japan 17, 980 (1962).

${ }^{27}$ N. Mikoshiba, Rev. Mod. Phys. 40, 833 (1968).

\title{
Luminescence of Donor-Acceptor Pairs in Cubic SiC
}

\author{
W. J. Choyke and Lyle Patrick \\ Westinghouse Research Laboratories, Pittsburgh, Pennsylvania 15235
}

(Received 1 July 1970)

\begin{abstract}
The photoluminescence of donor-acceptor pairs, under laser excitation, was recorded at $1.8^{\circ} \mathrm{K}$. The spectrum is type II, and is attributed to $\mathrm{N}$-Al pairs. Well-resolved lines were identified up to the 80th shell, and the van der Waals and multipole interactions were evaluated, following procedures used for the very similar GaP pair spectra. The limiting photon energy for distant pairs is $h \nu_{\infty}=2.0934 \mathrm{eV}$. As in some $\mathrm{GaP}$ spectra, intensity anomalies were observed. With the $\mathrm{GaP}$ work serving as a guide, one intensity anomaly was interpreted as a channel cutoff, another as a phonon resonance. This permitted us to evaluate the ionization energies, $E_{\mathrm{D}}=118 \mathrm{meV}$ for $\mathrm{N}$, and $E_{\mathrm{A}}=(179 \mathrm{meV})+E_{x}$ for $\mathrm{Al}$, with $E_{x}$ the still unknown exciton binding energy. With lower resolution the maximum and the half-width of the spectrum envelope were measured as a function of excitation intensity, and phonon replicas were recorded. The band maximum at low excitation yields an impurity level of $1.6 \times 10^{18} \mathrm{~cm}^{-3}$
\end{abstract}

\section{INTRODUCTION}

The low-temperature luminescence of donor-acceptor (DA) pairs yields a spectrum rich in narrow lines. ${ }^{1} \mathrm{DA}$ pairs in $\mathrm{GaP}$ provide the best and most numerous examples. The early GaP spectra gave information on donor and acceptor ionization energies, ${ }^{2}$ and on the van der Waals interaction between neutral donors and acceptors. ${ }^{3}$ Improvements in crystal growth and in experimental techniques yielded spectra of high quality that permitted a more detailed analysis. ${ }^{4,5}$ These spectra provided information on the multipole moments of the ions, ${ }^{6}$ and some details of the double-capture process that precedes light emission. ${ }^{7}$

The $\mathrm{SiC}$ spectrum we show here was first observed in 1963 , and attributed to N-Al pairs, ${ }^{8}$ but it appeared impossible to give a satisfactory analysis at that time. We now use laser excitation to obtain a spectrum comparable in quality to some of the $\mathrm{GaP}$ spectra, and we can use the experience gained in $\mathrm{GaP}$ to analyze it fully. Cubic $\mathrm{SiC}$ and $\mathrm{GaP}$ have the same crystal structure (zinc-blende), hence the same shell structure for DA pairs.

In Sec. II terms are defined, and theoretical relationships are summarized. The experimental results are shown in Sec. III. Our fitting of the spectrum is then discussed in Sec. IV. The interpretation of two intensity anomalies permits us to break down into separate parts the value deduced for the sum of the ionization energies, $E_{\mathrm{D}}+E_{\mathrm{A}}$. We discuss the multipole terms, and the difficulties encountered in fitting close pairs. Our conclusions are summarized in Sec. V.

\section{THEORETICAL RELATIONSHIPS}

The lines of a DA pair spectrum are fitted to the formula first given by Hopfield et al.,

$$
h \nu=E_{\mathrm{g}}-\left(E_{\mathrm{D}}+E_{\mathrm{A}}\right)-E_{\mathrm{C}}+E_{\mathrm{vdW}},
$$

where $E_{\mathrm{g}}$ is the energy gap, $E_{\mathrm{D}}$ and $E_{\mathrm{A}}$ are the donor and acceptor ionization energies, $E_{\mathrm{C}}$ is the Coulomb interaction energy between donor and acceptor ions after electron-hole recombination, and $E_{\mathrm{vdW}}$ is the interaction energy between neutral donor and acceptor atoms before recombination.

At first it was assumed that $E_{\mathrm{C}}=-e^{2} / \epsilon r$, where $\epsilon$ is the static dielectric constant and $r$ is the DA separation, which takes on the set of discrete values permitted by the lattice structure. Thus, for a given donor, a value of $E_{\mathrm{C}}$ was calculated for each shell of equidistant acceptors, the shells being denoted by the integers $m$. However, for nearest neighbors (small $m$ ) it was observed that spectral lines had to be assigned to each crystallographically inequivalent set of a given shell. It is now possible to account for the shell substructure by including multipole terms in $E_{\mathrm{C}}$, for these terms have a dependence on both the DA separation and lattice direction. ${ }^{6}$ 